\title{
Many strings to one bow: a multi-project approach by a Royal College of Physicians chief registrar
}

\author{
Author: Raunak Singh
}

\section{Aims}

As a Royal College of Physicians chief registrar (CR) at University Hospitals of Leicester (UHL) NHS Trust, multiple quality improvement opportunities presented themselves focusing on the betterment of junior doctors. I aim to highlight the benefits to an NHS trust in supporting a CR with a multi-project approach (MPA).

\section{Methods}

Five strands were identified as part of a MPA, selected through consideration of local needs, national areas of focus and personal interests.

\section{Results}

Junior doctor morale 'Listening into action' (LiA): An extensive survey with 402 junior doctor respondents (42.6\%) identified key themes: team working and relationships; feedback; training and education; resources; wellbeing and pastoral support; staffing and workload; senior clinician support; and autonomy. These have underpinned a high-powered LiA campaign, improving the working lives of junior doctors at UHL.

Medicine directorate induction: Frequent rotations, and a large directorate pose challenges in ensuring comprehensive induction. Fundamental improvements included CR involvement; streamlined agenda; concise and up-to-date presentations; attendee feedback; hand-outs; certification of induction; and providing lunch. The rating of 'quality of induction in preparing to work in the directorate' improved from $75 \%(n=28)$ rating induction as good or excellent, to $100 \%(n=21)$.

Exception reporting: Despite senior support of this important feature of the 2016 junior doctor contract, $91 \%$ of junior doctors surveyed $(n=74)$ across UHL identified barriers to exception reporting. To embed this culture change, improvements are targeting: improved access to reporting; changing a perceived fear culture to one of supportiveness; and clearer guidance and education.

JDFMed: Increasingly, junior doctors feel disconnected from senior clinical and non-clinical leadership. To bridge this gap,

Author: Royal College of Physicians chief registrar, University Hospitals of Leicester NHS Trust, Leicester, UK an informal junior doctor forum (JDFMed) for those working in medicine has been established to provide a safe place to air concerns, and provide a conduit to senior leadership. Challenges of maintaining attendance at such fora are perhaps a reflection of trainee workload and systemic disillusionment, highlighting the need to engage junior doctors.

Junior doctor of the month awards: Local and national literature highlights the importance of recognising excellence in improving training experience and satisfaction. Realising that a culture shift is needed to make positive feedback the norm, a monthly award mechanism in medicine has been established, with scope for expansion to other directorates at UHL.

\section{Conclusion}

If well-supported by their trusts, CRs have a unique opportunity to create positive impact in multiple areas rather than a traditional single-project approach. MPA highlights that having many strings to one bow can result in both directorate and trust-level improvements.

\section{Conflict of interest statement}

None. 